\title{
Producción cíclica de hidrógeno a partir de fracciones ácidas de bio-oil: ácido acético
}

\author{
Jaime Lachen, Jorge Plou, Paul Durán, Javier Herguido, José Ángel Peña \\ CREG (Grupo de Catálisis, Separaciones Moleculares e Ingeniería de Reactores) \\ Instituto de Investigación en Ingeniería de Aragón (I3A). \\ Universidad de Zaragoza, Mariano Esquillor s/n, 50018, Zaragoza, Spain. \\ Tel.+34-976762390, Fax +34-976762043, e-mail:jplou@unizar.es
}

\begin{abstract}
La combinación de los procesos de descomposición catalítica de fracciones del bio-oil y "steam-iron" es capaz de producir hidrógeno de forma cíclica. Durante la primera etapa se reduce un óxido métalico, y en la segunda se produce hidrógeno de muy alta pureza (producción+purificación de $\mathrm{H}_{2}$ ).
\end{abstract}

\section{Introducción}

La necesidad del cambio en el actual modelo energético unido a la reducción del efecto invernadero empuja a la utilización de fuentes de energía renovable (solar, hidráulica, eólica y biocombustibles), en detrimento de las tradicionales fósiles (petróleo, carbón y gas natural).

Los llamados "biocombustibles", son productos de origen biológico, que provienen en su mayor parte del aprovechamiento de residuos generados por la actividad humana (agrícola, ganadero o industrial).

El bio-oil, líquido procedente de la pirólisis rápida de biomasa, está compuesto por decenas de especies diferentes en proporciones variables dependiendo de la materia prima de la que se obtenga, y del tratamiento al que se le someta. No obstante, tal dispersión puede agruparse en cuatro fracciones principales: alcohólica, ácida, fenólica y acuosa. El presente estudio pretende determinar mediante la utilización de compuestos representativos de cada una de las fracciones, la viabilidad para la producción de hidrógeno mediante el método "steam-iron" [1]. Concretamente este trabajo aborda la producción a partir de la fracción ácida de bio-oil, utilizando el ácido acético como compuesto modelo, al tratarse del mayoritario [2].

El ciclo se compone de dos etapas. En la primera se produce la reducción del sólido (óxido de hierro con aditivos) a partir de los productos gaseosos provenientes de la descomposición térmica del ácido acético. Para favorecer tal descomposición, el óxido metálico se acompaña de un catalizador en pequeñas proporciones (10\%w). En la segunda etapa, la reoxidación del hierro con vapor de agua produce hidrógeno puro.

\section{Experimental}

Los experimentos han sido llevados a cabo en una planta a escala de laboratorio, compuesta por la zona de alimentación, zona de reacción y zona de análisis. La alimentación de gases se realiza mediante medidores de flujo másico de gas y la vaporización se realiza alimentando líquido con una bomba HPLC y un vaporizador con alta carga térmica. La reacción se lleva a cabo en un reactor de cuarzo alojado en un horno eléctrico. Los vapores de salida son condensados en una trampa fría (Peltier) y los gases resultantes analizados mediante $\mu$-GC.

El sólido es una mezcla de óxido de hierro dopado, aluminato de níquel (efecto catalítico) y sílice como diluyente sólido. El óxido de hierro es la especie reactiva y está compuesto por $\mathrm{Fe}_{2} \mathrm{O}_{3}, \mathrm{Al}_{2} \mathrm{O}_{3}$ y $\mathrm{CeO}_{2}$. La hematita es la fase mayoritaria $(98 \% \mathrm{w})$. Los otros dos óxidos actúan como estabilizadores de la estructura cristalina y como promotores de la reacción. Se ha utilizado un método sol-gel (citratos) para la obtención del sólido [3].

El catalizador es $\mathrm{NiAl}_{2} \mathrm{O}_{4}$ y se ha producido con un $10 \%$ w en exceso de níquel (en forma de $\mathrm{NiO}$ ). Durante la primera reducción el $\mathrm{NiO}$ se reduce a níquel metálico que actúa como especie catalítica activa. La síntesis se realiza mediante coprecipitación a pH ascendente [4].

\section{Resultados y discusión}

Se han realizado experimentos alimentando ácido acético al $10 \%$ v diluido en argón. La mezcla de sólidos está compuesta de un $67.5 \%$ w de óxido de hierro dopado y un $7.5 \%$ w de catalizador. Nótese que la cantidad final de fase activa de $\mathrm{Ni}$ es de tan solo un $0.75 \%$ w. Finalmente a ésta se le añade un $25 \%$ w de $\mathrm{SiO}_{2}$. Las temperaturas nominales de 
trabajo fueron desde $650{ }^{\circ} \mathrm{C}$ hasta $800{ }^{\circ} \mathrm{C}$ para las etapas de reducción.

En la Figura 1 se muestran los valores del ratio $[\mathrm{CO}] /\left([\mathrm{CO}]+\left[\mathrm{CO}_{2}\right]\right)$ a la salida del reactor. Estos valores son representativos de las reacciones que se producen durante las etapas de reducción, y que se justifican mediante los equilibrios termodinámicos entre las especies. Primeramente se produce el paso de $\mathrm{Fe}_{2} \mathrm{O}_{3}$ a $\mathrm{Fe}_{3} \mathrm{O}_{4}$ y $\mathrm{NiO}$ a $\mathrm{Ni}$ en los primeros 5 minutos. La presencia de fase activa de catalizador (Ni) favorece la descomposición completa de ac. acético. Los gases generados son mayoritariamente $\mathrm{CO}, \mathrm{CO}_{2}, \mathrm{H}_{2}$ y $\mathrm{H}_{2} \mathrm{O}$. A este primer paso le sucede la reducción de $\mathrm{Fe}_{3} \mathrm{O}_{4}$ a hierro metálico, caracterizado por un tramo de composiciones prácticamente constantes y duración variable dependiendo de la temperatura de operación. Los valores experimentales están ligeramente por encima del teórico (líneas punteadas), debido a que la reducción del sólido no es completa, y a que se produce un efecto de "dilución" por el lecho de sólido no reducido. Una vez éste completamente reducido, el comportamiento queda perfectamente descrito por la reacción de water gas shift (WGS).

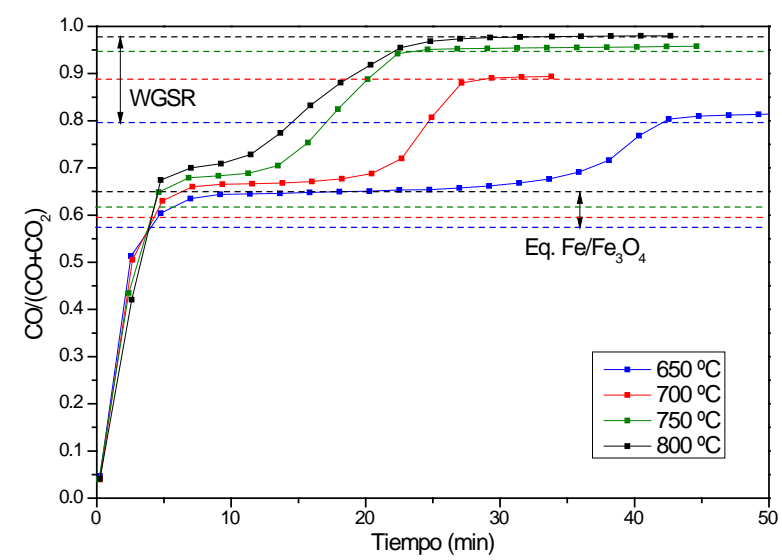

Figura 1. Reducción de hematita con ácido acético.

La etapa de oxidación se verifica siempre a una temperatura de $550^{\circ} \mathrm{C}$. Es una temperatura suficiente para poder oxidar el hierro sin necesidad de castigar térmicamente la mezcla. Ha sido escogida en base a estudios previos [5].

En la Figura 2 se muestran los valores de conversión de vapor de agua durante las oxidaciones. La diferencia de comportamiento, a pesar de trabajar a la misma temperatura, se debe a la historia térmica del sólido. Cuanto más tiempo ha pasado a temperaturas altas, menor conversión media se aprecia en las oxidaciones, y por tanto mayor es el tiempo para la reoxidación completa (hasta $\mathrm{Fe}_{3} \mathrm{O}_{4}$ ). No se observó presencia de $\mathrm{CO}$ ni $\mathrm{CO}_{2}$, por lo que la pureza del hidrógeno está asegurada hasta niveles cercanos al límite de detección del aparato $\mu$-GC.

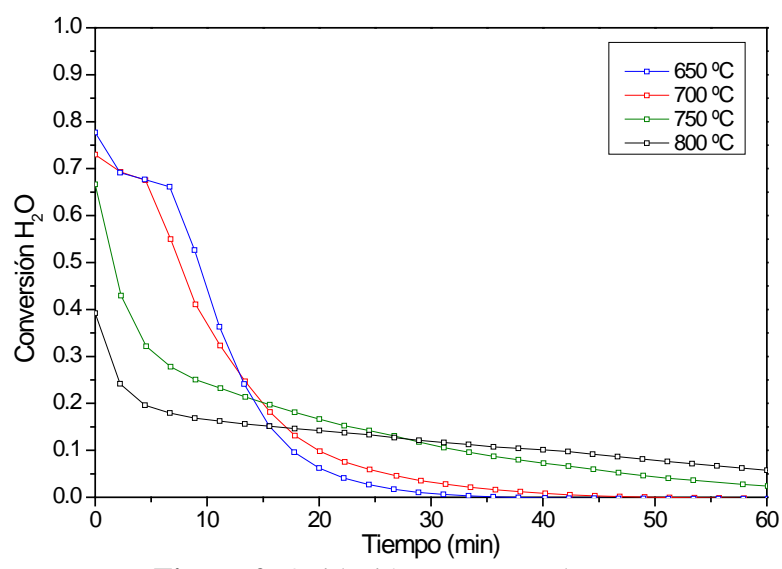

Figura 2. Oxidación con vapor de agua.

\section{Conclusiones}

Los experimentos realizados a diferentes temperaturas en la reducción han mostrado que el ácido acético, como componente modelo del bio-oil, es capaz de reducir el sólido a sus niveles de oxidación más bajos. Este hecho es fundamental para poder obtener hidrógeno puro en la posterior oxidación.

Las temperaturas de operación más adecuada (entre 700 y $750{ }^{\circ} \mathrm{C}$ ) es aquella para las que se se produce el mayor caudal de hidrógeno durante el menor periodo de tiempo, incluyendo tanto la etapa de reducción como la de oxidación.

\section{REFERENCIAS}

[1] A. Messerchmitt. Process of producing hydrogen. U.S. Patent 971,206 (1910).

[2] M.Bertero, G. de la Puente, U. Sedran. Fuel. 95 (2012) 263-271.

[3] E. Lorente, JA Peña, J. Herguido, Journal of Power Sources. 192 (2009) 224-229

[4] Al-Ubaid A., Wolf E.E.. Applied Catalalysis, 40 (1988).73-85.

[5] E. Lorente, JA Peña, J. Herguido. International Journal of Hydrogen Energy 33, (2008). 615-626. 